\title{
Post-operative colocolic intussusception
}

Accepted: 15 April 2002 / Published online: 9 April 2003

(C) Springer-Verlag 2003

\begin{abstract}
A 2-year-old boy presented with bowel obstruction 5 days after repair of a recurrent epigastric hernia. Pre-operative imaging implicated intussusception as a cause of the obstruction. At operation a gangrenous transverse colocolic intussusception was resected en masse. No clear lead point was seen. Recovery was uneventful.
\end{abstract}

Keywords Intussusception C Colocoli ·

Post-operative $\cdot$ Bowel Obstruction

\section{Introduction}

Post-Operative intussusceptions and colocolic intussusceptions are both rare entities. To our knowledge, the occurrence of a post-operative colocolic intussusception has not been previously reported. We present such a case, along with a review of pertinent literature.

\section{Case report}

A healthy boy with a history of functional constipation underwent repair of a $1-\mathrm{cm}$ epigastric hernia at age 23 months. A recurrence was diagnosed shortly after the procedure. The recurrent hernia was repaired electively at age 28 months under general anesthesia administered by laryngeal mask. The surgeon's impression was that the recurrence occurred due to failure of the previous sutures. The fascial defect was again closed with interrupted 000 Vicryl without apparent entry into the peritoneum. Total operative time was $25 \mathrm{~min}$.

S. Emil $\cdot$ X. Shaw $\cdot$ J.M. Laberge $(\square)$

Division of Pediatric General Surgery,

Department of Surgery,

Montreal Children's Hospital,

McGill University Health Center,

2300 Tupper Street,

C-1137, Montreal, Quebec,

H3H 1P3, Canada

E-mail: jean-martin.Iaberge@muhc.mcgill.ca
The child started having intermittent non-bilious vomiting on the 1st postoperative day. His symptoms waxed and waned over the following days. On the 4th post-operative day, he presented to the emergency department with non-bilious vomiting and diarrhea. On examination, the pharynx was red, the abdomen was soft and the wound appeared normal. Abdominal radiographs were not obtained. He was hydrated and discharged with a diagnosis of acute gastroenteritis. Over the next $24 \mathrm{~h}$ he worsened considerably, developing protracted vomiting, obstipation, and abdominal distention. He returned to the emergency department, where a surgical consultation was obtained.

Examination showed a dehydrated child in obvious discomfort. The abdomen was markedly distended and tympanitic. Highpitched bowel sounds were present. A right upper quadrant fullness was palpated. There was no evidence of peritonitis. Rectal examination stimulated the child to pass a moderate amount of bloody mucus. The WBC count was 11.4 with $12 \%$ band forms.

The abdomen was imaged with plain radiographs and ultrasound (Fig. 1). Multiple distended small bowel loops were seen. Ultrasound revealed an echogenic, heterogeneous mass in the right flank measuring $6.4 \mathrm{~cm}$ with a small anechoic center, consistent with intussusception. A presumptive diagnosis of small bowel intussusception was made and the child was prepared for surgery.

Exploration through a right lower quadrant trans-rectus incision revealed a colocolic intussusception of the transverse colon (Fig. 2). The mass was tense and gangrenous, but not perforated. No attempt at reduction was made. The mass was removed en bloc and bowel continuity restored with an end-end sutured anastomosis. Pathology revealed hemorrhage between the serosa and muscularis of the intussusceptum, but no lead points. The child recovered uneventfully and was discharged 5 days after operation. He continues to do well 2 years after the episode.

\section{Discussion}

Although rare, intussusception is an important cause of small bowel obstruction after abdominal surgery [1-4]. It has been estimated to occur in $0.08 \%$ to $0.5 \%$ of all pediatric laparotomies, [1, 2]. The typical patient is a child who develops symptoms of bowel obstruction after an initial return of bowel function. Two pediatric series by Holcomb et al. [1] and Ein and Ferguson [2] reported its occurrence only after major abdominal operations, including some that did not involve significant manipulation of the small or large bowel, such as Nissen 

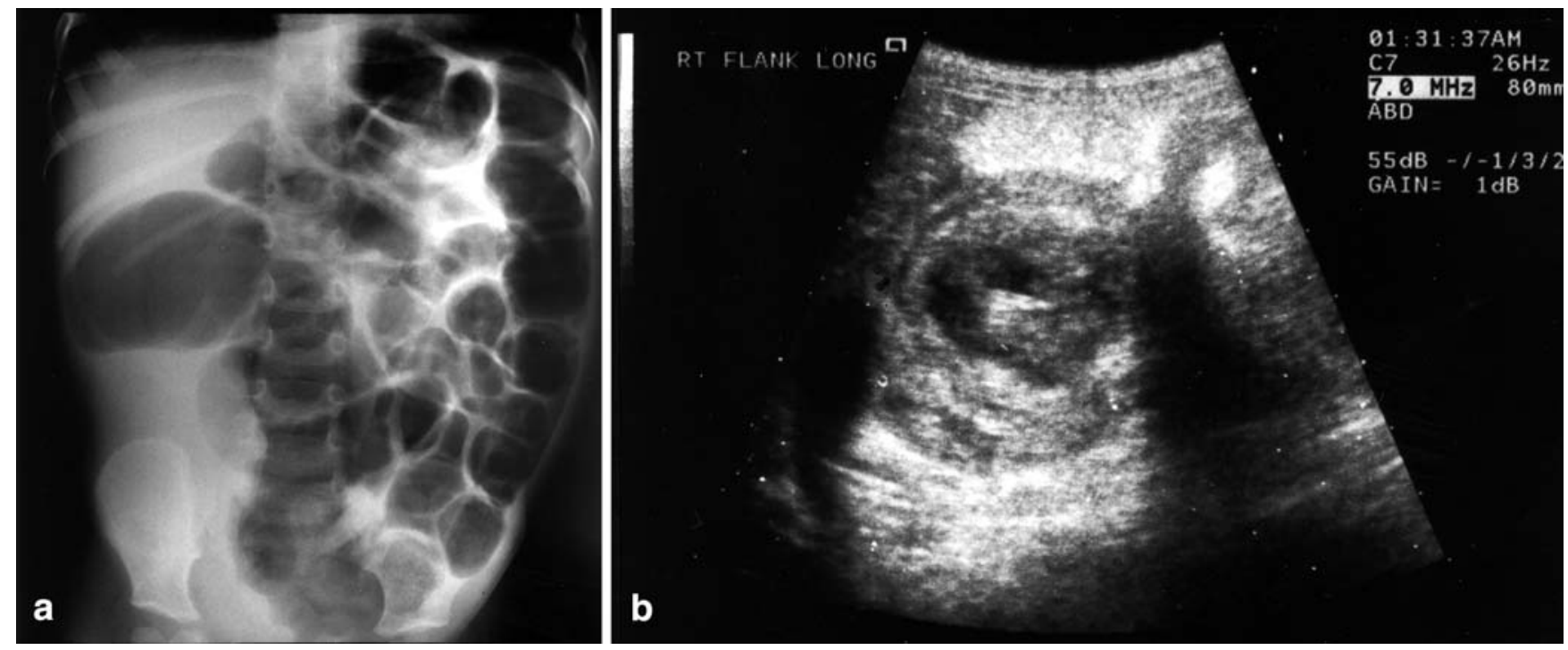

Fig. 1 Diagnostic imaging shows a multiple distended small bowel loops with a mass effect in the right flank and $\mathbf{b}$ a typical "target" sign on ultrasound

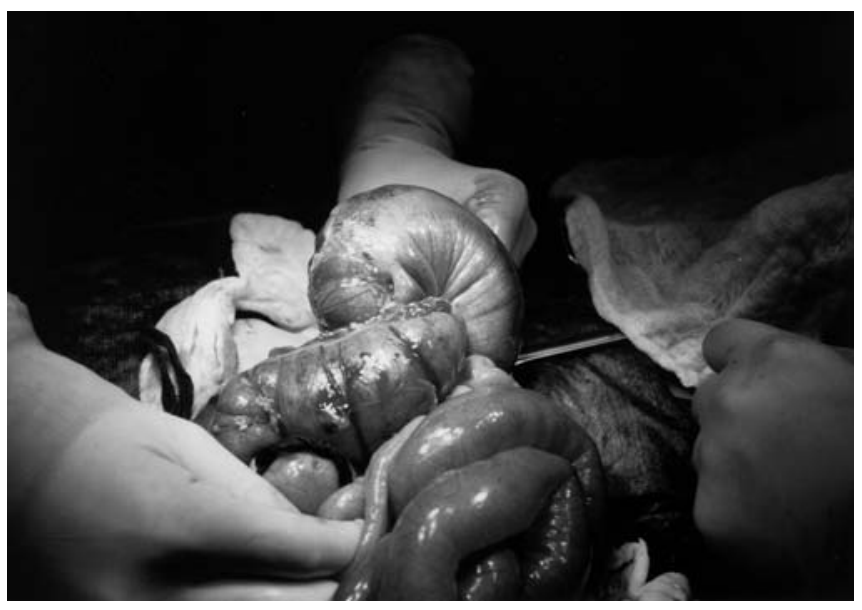

Fig. 2 Gross appearance of colocolic intussusception at laparotomy

fundoplication. More recently, Allbery et al. reported two cases after negative trauma laparotomy [3].The cases reported in the literature are overwhelmingly small bowel intussusceptions with a few ileocolic intussusception [1-4]. Our Medline search revealed no prior reports of post-operative intussusceptions that involved only the colon or that followed minor extra-peritoneal abdominal procedures, such as occurred in our patient.

Pediatric colocolic intussusceptions are also exceedingly rare [5-8]. Pneumatosis intestinalis precipitated a colocolic intussusception in an infant with severe Crohn's disease [5]. Colonic polyps [6] and bezoars [7] have acted as lead points for colocolic intussusceptions. Lipomas are reported as lead points for colocolic intussusceptions in adults [8].
We speculated on possible etiologies for this intussusception. Although not unusual in long-standing intussusception, hemorrhage in the wall of the intussusceptum led us to wonder whether a colonic hematoma may have been caused by an inadvertent needle stick during the hernia repair, or whether an epiploic appendix may have been present in the hernia and bled post-operatively. The patient's constipation may have also allowed a fecaloma to serve as a lead point. However, there was no proof that the peritoneum in the area of the epigastric hernia was violated and no impacted stool was seen within, or in the vicinity of, the intussusception. In the absence of a proven lead point, we have to think that this could also be an idiopathic post-operative colocolic intussusception, or even totally unrelated to the operation. ception was difficult to make and required a high index of suspicion. Recent reports emphasize the important role of ultrasound in making this diagnosis [3, 4]. Ultrasound will typically reveal the "target", "doughnut", or "pseudokidney" signs typical of any intussusception. This test should be used liberally in the clinical setting of post-operative bowel obstruction or unexplained postoperative abdominal pain and vomiting.

\section{References}

1. Holcomb GW 111, Ross AJ Ill, O’Neill JA Jr (1991) Postoperative intussusception: increasing frequency or increasing awareness? South Med J 84:1334-1339

2. Ein SH, Ferguson JM (1982) Intussusception-the forgotten postoperative obstruction. Arch Dis Child 57:788-790

3. Allbery SM, Swischuk LE, John SD, Angel C (1998) Postoperative intussusception: often an elusive diagnosis. Pediatr Radiol 28:271

4. Carnevale E, Graziani M, Fasanelli S (1994) Post-operative ileoileal intussusception: sonographic approach. Pediatr Radiol 24:161-163
In the past, the diagnosis of post-operative intussus- 
5. Dubinsky MC, Deslandres C, Patriquin H, Seidman EG (2000) Pneumatosis intestinalis and colocolic intussusception complicating Crohn's disease. J Pediatr Gastroenterol Nutr 30:96-98

6. Arthur AL, Garvey P, Vaness DG (1990) Colocolic intussusception in a three-year-old child caused by a colonic polyp. Connecticut Med 54:492-494
7. Fildes RD, Springate JE, Jewett T, O’Shea MM, Feld LG (1989) Colonic intussusception secondary to a calcium phosphate bezoar in a child receiving calcium carbonate for hyperphosphatemia. J Pediatr Surg 24:1195-1197

8. Zamboni WA FleisherH, ZanderJD, FolseJR(1987)Spontaneous expulsionof lipoma per rectum occurring with colonic intussusception. Surgery 101:104-107 